\title{
Proangiogenic cells enhanced persistent and physiologic neovascularization compared with macrophages
}

\author{
Young-Eun Choi ${ }^{1}$, Young Ryun $\mathrm{Cha}^{1}$, Kyoung-min Lee, Hyun Jin Kim and Chang-Hwan Yoon
}

Proangiogenic cells (PACs) display surface markers and secrete angiogenic factors similar to those used by myelomonocytic cells, but, unlike myelomonocytic cells, PACs enhance neovascularization activity in experimental ischemic diseases. This study was performed to reveal the differential neovascularization activities of PACs compared with those of myelomonocytic cells. We cultured PACs and CD14+-derived macrophages (Macs) for 7 days. Most of the surface markers and cytokines in the two cell types were alike; the exceptions were KDR, $\beta 8$ integrin, interleukin-8 and monocyte chemotactic protein-1. Unlike Macs, PACs significantly enhanced mesenchymal stem cell (MSC) transmigration. PACs and Macs increased neovascularization activity in an in vitro co-culture of human umbilical vein endothelial cells and MSCs and in an in vivo cotransplantation in Matrigel. However, the use of Macs resulted in inappropriately dilated and leaky vessels, whereas the use of PACs did not. We induced critical hindlimb ischemia in nude mice, and then transplanted PACs, Macs or vehicle into the mice. We obtained laser Doppler perfusion images weekly. At 2 weeks, mice treated with PACs showed significantly enhanced perfusion recovery in contrast to those treated with Macs. After day 7, when cells were depleted using a suicidal gene, viral thymidine kinase, to induce apoptosis of the cells in vivo by ganciclovir administration, we found that the improved perfusion was significantly abrogated in the PAC-treated group, whereas perfusion was not changed in the Mac-treated group. PACs caused an increase in healthy new vessels in in vitro and in vivo models of angiogenesis and enhanced long-term functional neovascularization activity in the hindlimb ischemia model, whereas Macs did not. Nevertheless, the angiogenic potential and long-term functional results for a specific cell type should be validated to confirm effectiveness and safety of the cell type for use in therapeutic angiogenesis procedures.

Experimental \& Molecular Medicine (2015) 47, e186; doi:10.1038/emm.2015.60; published online 25 September 2015

\section{INTRODUCTION}

Since the initial discovery of adult $\mathrm{CD}^{+} 4^{+}$cell-derived endothelial progenitor cells (EPCs) by Asahara et al., ${ }^{1}$ various groups have studied the origin and the therapeutic benefits of EPCs. Several subsets of $\mathrm{CD} 34^{+}$or $\mathrm{CD} 133^{+}$cells were reported to have the potential to home in on the ischemic region and enhance neovascularization activity in experimental or clinical trials. ${ }^{1-3}$ Because putative EPCs are very rare in adult peripheral blood, some investigators cultured adult peripheral blood derived-mononuclear cells to expand proangiogenic cell populations ex vivo. ${ }^{4-6}$ The culture conditions and the duration of the culturing period affected the characteristics of the resultant cells. After a short culture period of 4-7 days for peripheral blood-derived mononuclear cells, the so-called 'early EPCs' expressed monocytic and hematopoietic surface markers; therefore, they were called proangiogenic macrophages (Macs) or proangiogenic cells (PACs), respectively. ${ }^{7-9}$ Although PACs showed rare endothelial differentiation, they were preferentially localized to perivascular spaces in animal models. ${ }^{10,11}$ Despite very limited endothelial differentiation, PACs reproducibly increased neovascularization, ${ }^{12,13}$ and paracrine mechanisms were suggested to mediate the neovascularization improvement by PACs. However, cytokines from PACs are thought to be effective only in an early phase of neovascularization. ${ }^{14} \mathrm{We}$ recently demonstrated that the late (>1-2 weeks) depletion of injected PACs by means of a suicide gene hinders perfusion in hindlimb ischemia and acute myocardial infarction models. ${ }^{15,16}$ This loss of function within weeks after the in vivo injection of the cells suggests that PACs persist in the ischemic tissue and also contribute to the late phase of neovascularization. Because

\footnotetext{
Cardiovascular Center, Seoul National University Bundang Hospital, Seongnam, Republic of Korea

${ }^{1}$ These authors contributed equally to this work.

Correspondence: Dr C-H Yoon, Department of Internal Medicine, Seoul National University Bundang Hospital, 82 Gumiro 173, Bundang, Seongnam 436-707, Republic of Korea. 
the physical contribution of PACs to the endothelial monolayer is very limited, one may speculate that the perivascularlocalized cells may have contributed to the effect. However, the exact mechanism of the late-term effect is unclear.

On the other hand, macrophage colony-stimulating factor (M-CSF) stimulates the in vitro proliferation and differentiation of monocytes into mature authentic Macs, demonstrating antimicrobial capabilities by the production of cytokines and peroxide. ${ }^{17}$ Monocytes, or Macs, have shown contradictory results regarding whether they enhance arteriogenesis or neovascularization. ${ }^{12,18-20}$ Because PACs share many characteristics with Macs, ${ }^{8}$ we compared the angiogenic properties of PACs with those of authentic Macs in different experimental models of angiogenesis to gain further insights into the mechanisms of PAC-mediated angiogenesis.

\section{MATERIALS AND METHODS Cells}

The protocol was approved by the ethical committee of the Seoul National University Bundang Hospital (institutional review board no. B-1111-068-011). Written informed consent was provided by each donor. Peripheral blood mononuclear cells from healthy volunteers were isolated by density gradient centrifugation with Ficoll-Paque plus (GE Healthcare, Uppsala, Sweden). To obtain ex vivo expanded PACs, $8 \times 10^{6}$ peripheral blood mononuclear cells per ml medium were plated on culture flasks coated with human fibronectin (Sigma, Seoul, Korea) and maintained in EGM medium (endothelial basal medium (Lonza, Basel, Switzerland) supplemented with EGM SingleQuots) and $20 \%$ fetal bovine serum (FBS, Life Technologies, Seoul, Korea). After 3 days in culture, nonadherent cells were removed by thorough washing with phosphate-buffered saline (PBS), and adherent cells were used for viral transduction.

Pooled human umbilical vein endothelial cells (HUVECs) were purchased from InnoPharmaScreen (Chungnam, Korea) and cultured in EGM medium. Human mesenchymal stem cells (MSCs, Lonza) were purchased and cultured in Dulbecco's modified Eagle's medium containing 10\% FBS. HEK 293 cells were maintained in Dulbecco's modified Eagle's medium containing $10 \%$ FBS. CD14 ${ }^{+}$cells were isolated from peripheral blood by magnetic beads (Miltenyi Biotech, Seoul, Korea) and were cultured on a noncoated plate in RPMI medium (Life Technologies) supplemented with M-CSF $\left(50 \mathrm{ng} \mathrm{ml}^{-1}\right)$ and 10\% FBS for 7 days; these cells were used as macrophages.

\section{Preparation of lentiviral stocks}

Self-inactivating lentiviral vectors containing the enhanced green fluorescent protein (eGFP) gene or the viral thymidine kinase gene from herpes simplex type II virus and a WPRE (woodchuck posttranscriptional regulatory element) were generated by transient transfection in $293 \mathrm{~T}$ cells. The packaging plasmid used here was pCMV8.91, and pMD.G was used for vesicular stomatitis virus-G protein pseudotyping as previously described. ${ }^{16} \mathrm{After} 4 \mathrm{~h}$, the medium was replaced by EGM medium with EGM SingleQuots and 20\% FBS for $24 \mathrm{~h}$. Lentiviral particles were collected every $24 \mathrm{~h}$ for 3 days and were filtered through $0.22 \mu \mathrm{m}$ filters.

\section{Lentiviral transduction}

Lentiviral transduction in PACs, HUVECs, Macs and MSCs was carried out by adding viral supernatant to the specific media. After $24 \mathrm{~h}$, the media were changed, and a second transduction was performed. Transduced cells were used after three washes with PBS for the following experiments.

\section{Cytokine array}

After PBS washing, PACs and Macs were incubated with EGM medium without serum for 1 day. The supernatant was harvested the next day and was then used for cytokine arrays and multiplex enzyme-linked immunosorbent assays (Quantikine angiogenesis array, Ray Biotech, Norcross, GA, USA) according to the manufacturer's instructions. Briefly, cytokine array membranes were blocked in $2 \mathrm{ml}$ of $1 \times$ blocking buffer for $30 \mathrm{~min}$ and then incubated with $1 \mathrm{ml}$ of samples at room temperature for $1 \mathrm{~h}$. Samples were then decanted from each container, and the membranes were washed five times with $2 \mathrm{ml}$ of wash buffer at room temperature with shaking. Membranes were incubated in 1:250 biotin-conjugated primary antibodies at room temperature for $1 \mathrm{~h}$ and washed as described above, followed by an incubation in 1:1000 horseradish peroxidase-conjugated streptavidin for $60 \mathrm{~min}$. After this incubation, membranes were washed thoroughly and exposed to a peroxidase substrate for $5 \mathrm{~min}$ in the dark before imaging. Membranes were exposed to X-ray film (Kodak X-OMAT AR film, Rochester, NY, USA) for $1 \mathrm{~min}$. Signal intensities were quantified with a Bio-Rad VersaDoc Imaging System 3000 and analyzed with Quantity One software (Bio-Rad, Seoul, Korea). For each spot on the membrane, the net optical density level was determined by subtracting the background optical level from the total raw optical density level.

\section{Quantification of cytokines by multiplex immunoassays}

The concentrations of angiogenic cytokines monocyte chemotactic protein-1 (MCP-1), hepatocyte growth factor, interleukin-8 (IL-8), IL-17, basic fibroblast growth factor, platelet-derived growth factor-bb, vascular endothelial growth factor, IL-10 and tumor necrosis factor- $\alpha$ were measured by Bio-Plex Pro assays (Bio-Rad). These are multiplex immunoassays based on xMAP technology that was developed by Luminex (Austin, TX, USA). An antibody specific to each cytokine is covalently coupled to microspheres, with each antibody coupled to a different magnetic (MagPlex, Luminex) microsphere uniquely labeled with a fluorescent dye. The microspheres are incubated with standards, controls and samples in a 96-well microtiter plate at 300 r.p.m. for $30 \mathrm{~min}$ at room temperature. After washing with assay wash buffer, diluted biotinylated secondary antibody was added to the appropriate wells and incubated at 300 r.p.m. for $30 \mathrm{~min}$. After washing, streptavidin-phycoerythrin was added to each well and incubated for $10 \mathrm{~min}$. After a final wash, the plate was analyzed using the BioPlex 200 analyzer (Bio-Rad) to determine the concentration of the cytokines. The detection range of the standards is $\sim 1-10000 \mathrm{pg} \mathrm{ml}^{-1}$.

\section{Ganciclovir dose used to deplete target cells}

We used $500 \mu \mathrm{m}$ gancyclovir (GCV) for 5 days to deplete the thymidin kinase (TK)-transduced cells in vitro, and $100 \mathrm{mg} \mathrm{kg}^{-1} \mathrm{GCV}$ was injected intraperitoneally to deplete the cells in vivo beginning 7 days after cell transplantation.

\section{Two-dimensional pseudovascular tube formation assay}

We used an in vitro angiogenesis model to compare the effects of the presence of PACs or Macs on angiogenesis, as we have previously described. ${ }^{21}$ We cocultured MSCs that had been transduced with cyan fluorescent protein-expressing lentivirus and HUVECs that had been transduced with yellow fluorescent protein-expressing lentivirus in the presence of either PACs or Macs in EGM medium with 10\% FBS on a 
a
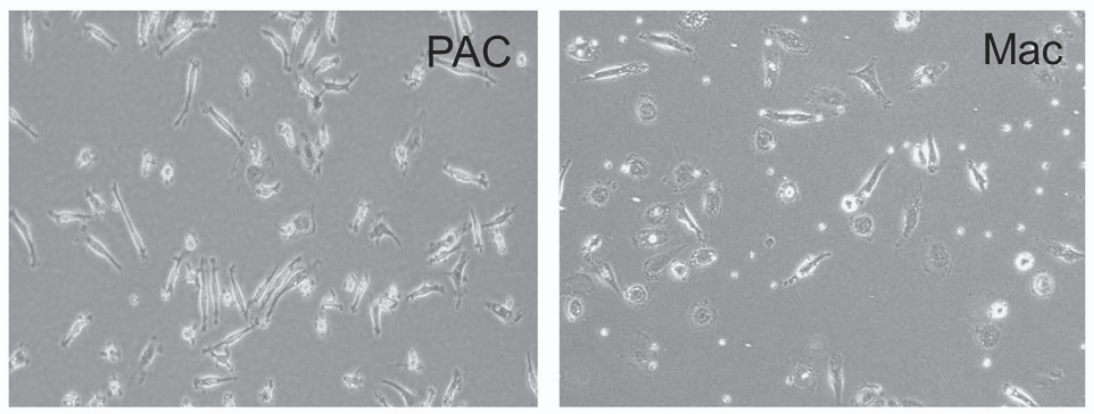

b
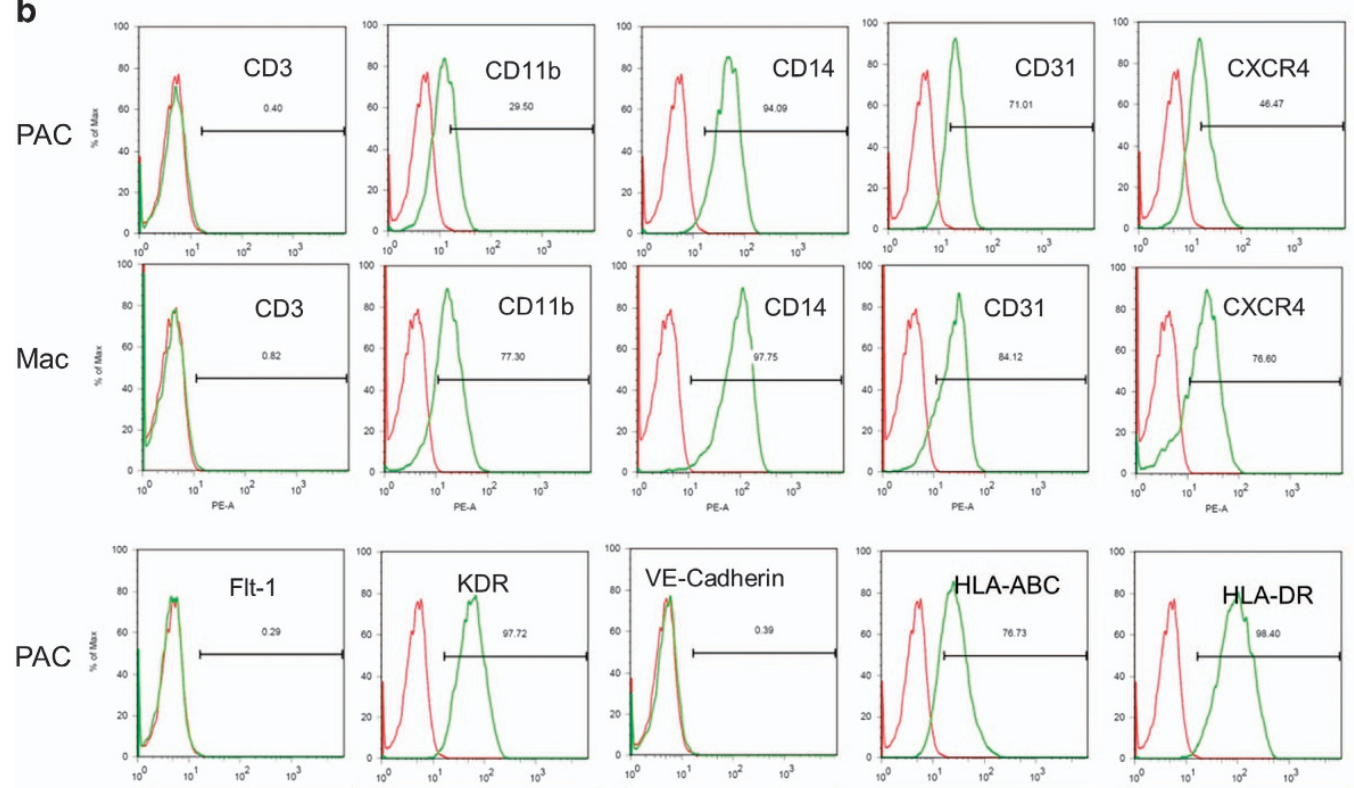

Mac
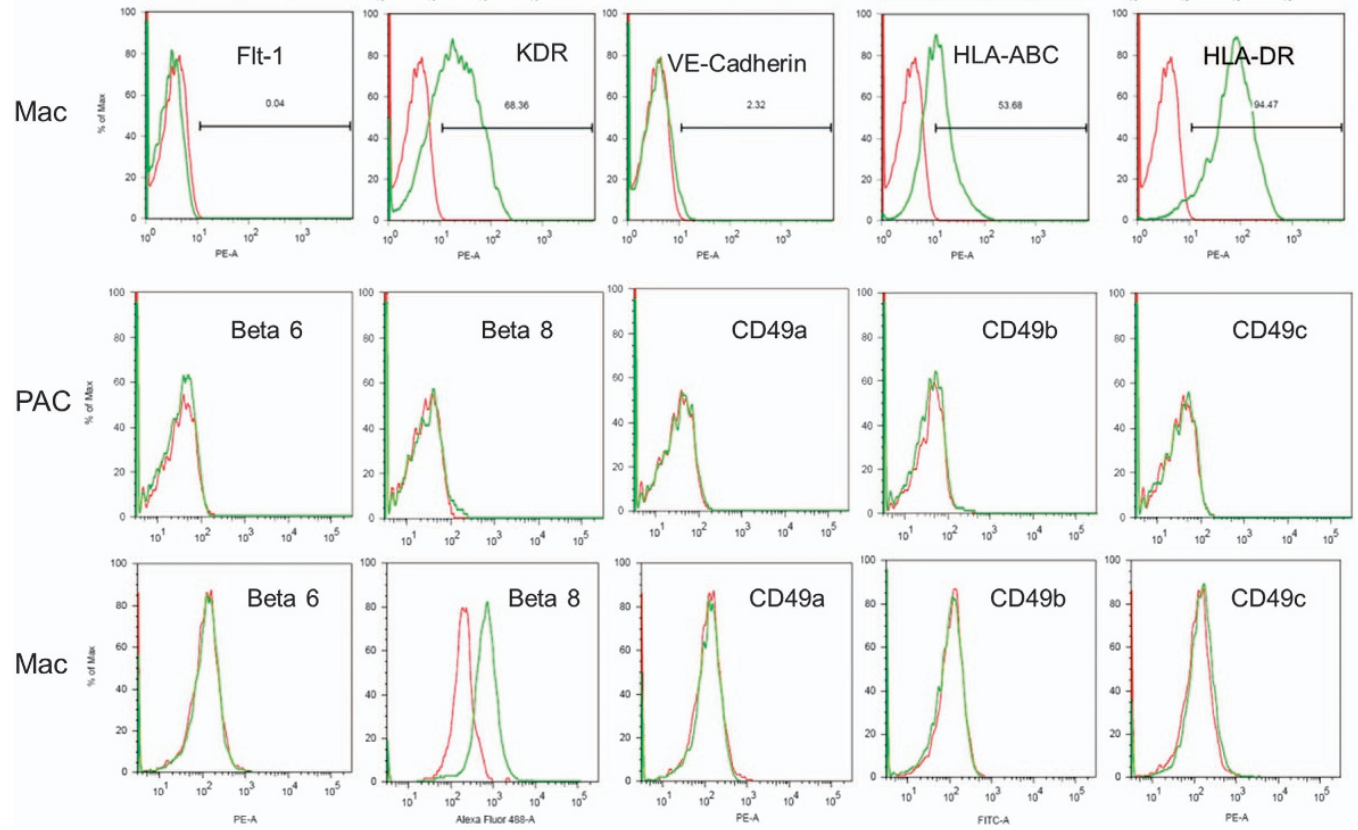

Figure 1 Comparison of surface markers and cytokine expression in proangiogenic cells (PACs) and macrophages (Macs). (a) Morphology of PACs and Macs in culture. PACs are long spindles and small, round cells, whereas Macs are short spindles and large, round and flat cells. (b) PACs and Macs similarly expressed CD11b and CD14; however, PACs showed higher KDR expression, and Macs showed higher integrin $\beta 8$ expression. (c) Cytokine array of the conditioned media for both types of cells. PACs secreted more interleukin-8 (IL-8) and less angiogenin and monocyte chemotactic protein-1 (MCP-1). PACs also secreted more angiopoietin-2, more angiostatin and different matrix metalloproteinases (*statistical significance, $P<0.05$ ). (d) Multiplex immunoassay revealed a significantly higher level of IL-8 and a lower level of MCP-1 in PAC cultures than in Mac cultures ( $P=0.032, n=6$ and $P=0.008, n=8$, respectively, paired $t$-test). 

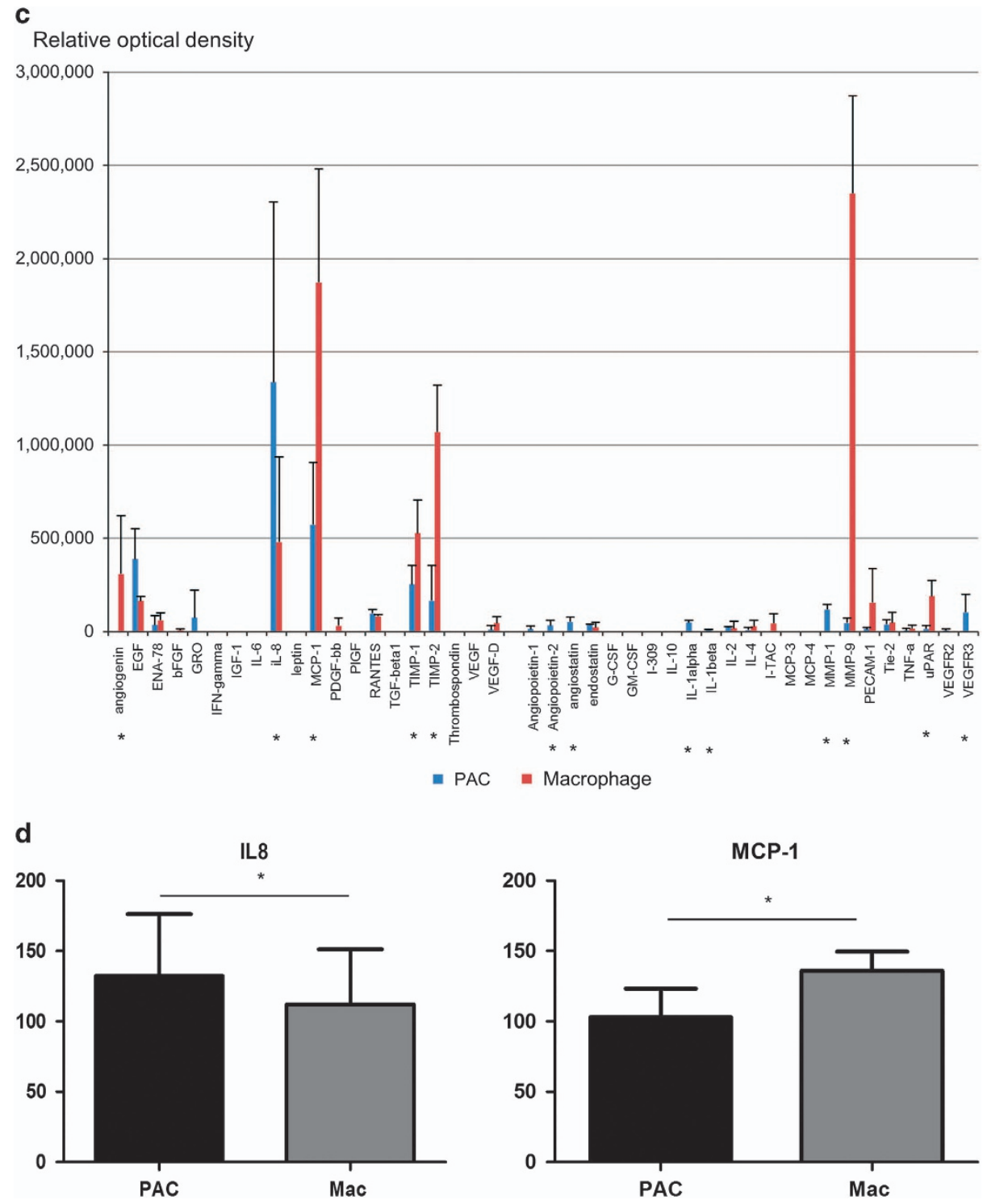

Figure 1 Continued

fibronectin-coated plate. We covered the cells with $200 \mu \mathrm{l}$ growth factor-reduced Matrigel (BD Biosciences, Seoul, Korea) in each well on 12-well plates. Images were taken after 7 days of culture.

\section{MSC transmigration assay}

We seeded $6 \times 10^{4}$ MSCs in each well of a 24 -well plate. At 1 day after seeding, we covered the MSCs with $250 \mu$ l growth factor-reduced Matrigel on ice. After $30 \mathrm{~min}$ of incubation at $37^{\circ} \mathrm{C}$, a control containing no cells or cultures containing PACs or Macs was seeded on the Matrigel. We quantified the total lengths of MSC filopodia that transmigrated upward through the Matrigel.

\section{Modified in vivo Matrigel plug model}

All procedures were approved by and performed in accordance with the Institutional Animal Care and Use Committee. MSCs and yellow fluorescent protein-labeled HUVECs that had been cultured for 7 days with overlying Matrigel, as described above, were harvested by cell scraper and then transplanted subcutaneously through lateral skin incisions on the backs of 8-week-old female athymic nude mice
(ORIENT, Seongnam, Korea). After 7 or 14 days, blood vessel growth into the Matrigel plugs was visualized by pre-mortem BS-1 lectin infusion $(200 \mu \mathrm{g}$, intravenous).

\section{Hindlimb ischemia model}

Eight-week-old male Balb/c nude mice (ORIENT) were anesthetized with isoflurane. The right femoral artery and vein were coagulated and then cut out to induce critical ischemia. The first laser Doppler perfusion image was taken 1 day after the operation at the time of cell administration via the tail vein. We injected $5 \times 10^{6}$ PACs or Macs into each animal. On days 7, 14 and 21, sequential laser Doppler perfusion images were taken. Perfusion to each limb was calculated from the mean value multiplied by the number of valid pixels in the region below the inguinal ligament. Data were represented as the ratio of the ischemic limb perfusion to that of the nonischemic limb.

\section{Immunostainings}

At $30 \mathrm{~min}$ before killing mice, $200 \mu \mathrm{g}$ TRITC-labeled BS-1 lectin (Sigma) was administered intravenously to stain perfused vessels. 

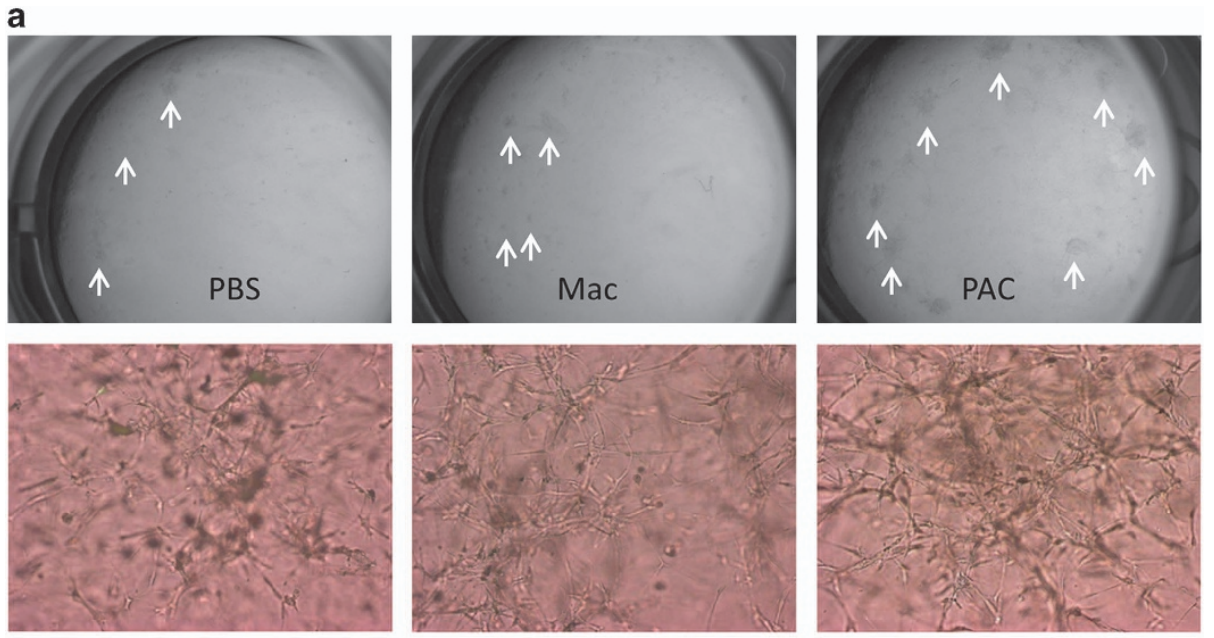

b

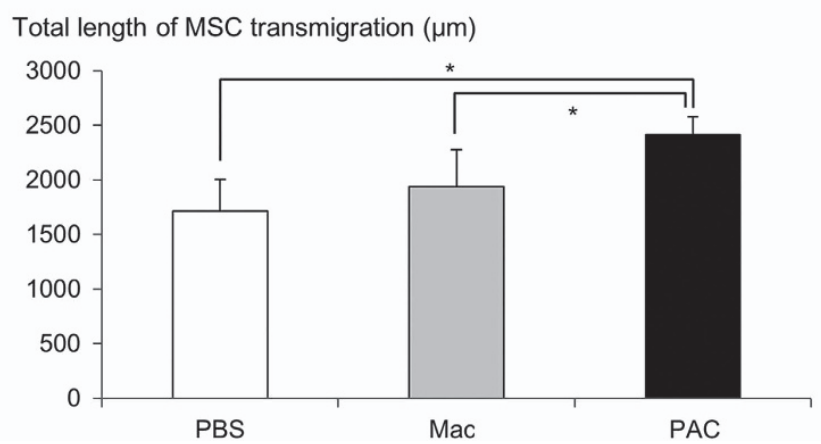

Figure 2 Proangiogenic cells (PACs) recruit mesenchymal stem cells (MSCs) more efficiently than do macrophages (Macs). (a) PACs induced the plaque formation of transmigrated MSCs in three-dimensional (3D) Matrigel. Arrows (upper panel) indicate the distinct morphology of MSC plaques. MSCs appeared as multilayered web-like networks (lower panel). (b) The presence of PACs significantly increased the transmigration of MSCs ( $P=0.0003$ by analysis of variance (ANOVA); $n=15,16$ and 15, respectively; post hoc analysis: Mac vs PAC—significant, phosphate-buffered saline (PBS) vs PAC—significant). * denotes statistical significance, $P<0.05$.

To create frozen sections, tissues or in vivo Matrigel were immersed in optimum cutting temperature compound and then frozen at $-80^{\circ} \mathrm{C}$. Frozen tissues were cut by cryotome to a thickness of 10 to $50 \mu \mathrm{m}$. The following antibodies were used in overnight incubations: fluorescein isothiocyanate-labeled antibody against $\alpha$-smooth muscle actin (Sigma) for hindlimb muscle and TRITC-labeled antibody against $\alpha$-smooth muscle actin (Sigma) for in vivo Matrigel. Tissues were imaged on a 510 META confocal laser scanning microscope (Zeiss, Jena, Germany). Samples were excited with 405, 458, 488, 514, 543 or $633 \mathrm{~nm}$ laser lines with a BP420-480, LP505, BP560-615 and LP650 chroma filter set or a variable spectral setting with a meta-detector using Plan-Neofluar objectives $(20 \times / 0.5$ or $40 \times / 1.3$ Oil). Threedimensional stacks of the tissues were taken at $2-4 \mu \mathrm{m}$ intervals spanning whole GFP $(+)$ cells $(20-40 \mu \mathrm{m})$. The pinhole settings were at 2.0 airy units. The $Z$-stack images were reconstructed as three-dimensional images or reformatted by projection view using Zeiss LSM image software.

\section{Capillary density}

Capillary density was quantified using the three-dimensional reconstructed images from $30 \mu \mathrm{m}$-thick hindlimb muscle tissue. In the muscle tissue, we quantified the number of capillaries that run along the muscle fibers through a random vertical plane to the muscle fiber.

\section{Statistics}

Continuous variables are represented as the mean \pm s.e. SPSS 20.0 software (IBM, Armonk, NY, USA) was used for statistical analysis of the data. Mean data were analyzed by Mann-Whitney tests between two groups and one-way analysis of variance or Kruskal-Wallis tests followed by post hoc analysis by Bonferroni for more than three groups.

\section{RESULTS}

PACs express and secrete different cytokines than do Macs The cultured PACs were spindle-shaped cells mixed with small round cells, and MACs were large, round, tightly attached cells mixed with spindle-shaped cells (Figure 1a). Most of the expressed surface markers, such as CD11b and CD14, were very similar between PACs and Macs, except for differences in KDR and integrin $\beta 8$ expression levels (Figure 1b).

PACs and Macs secreted different profiles of cytokines and matrix metalloproteinases related to angiogenesis, as detected by a cytokine array (Figure 1c). For example, PACs secreted more IL- 8 but less MCP-1 than did Macs. A multiplex immunoassay also revealed higher levels of IL-8 and lower levels of MCP-1 in PACs than in Macs (Figure 1d). Hepatocyte 


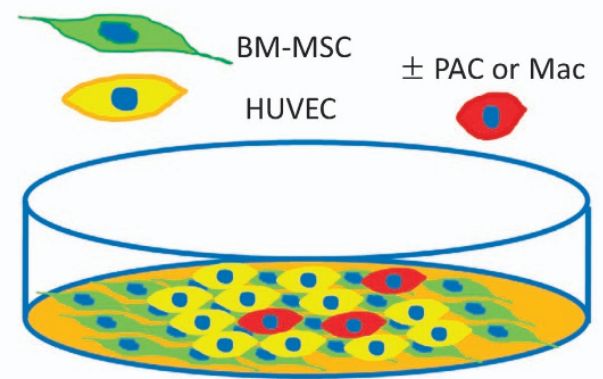

b
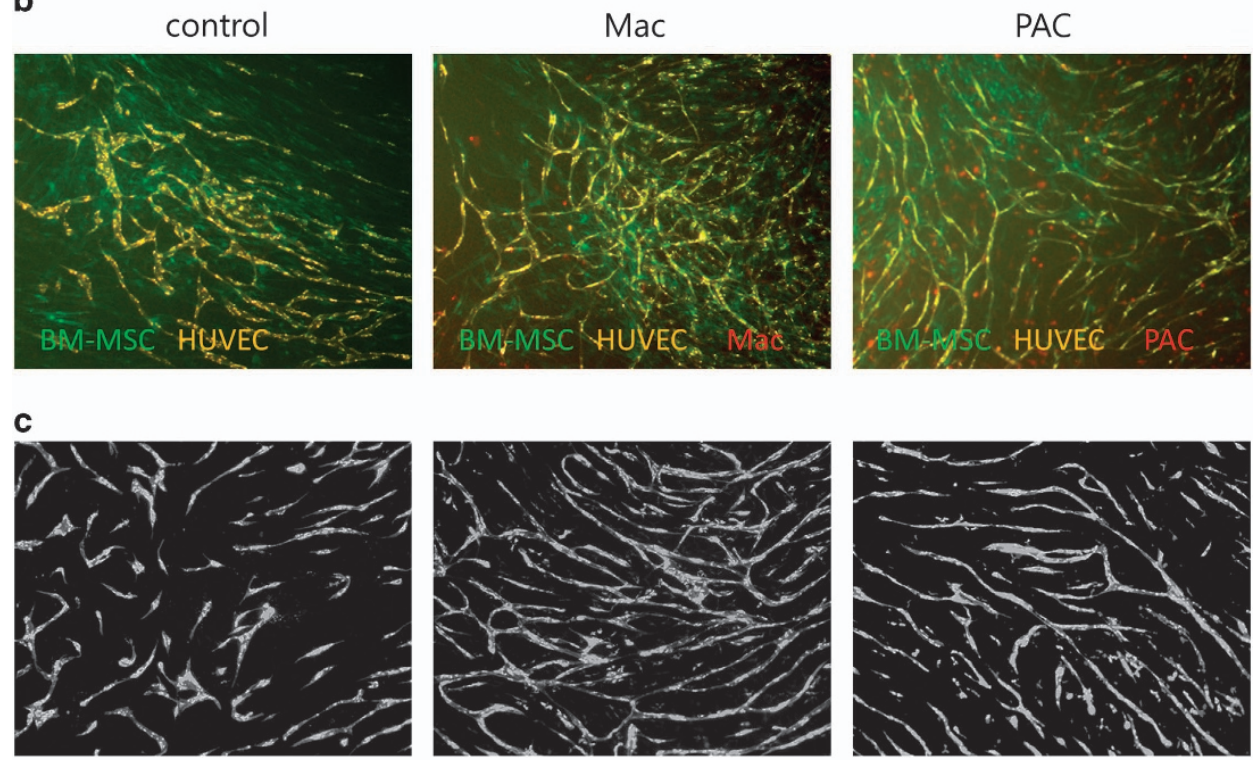

d

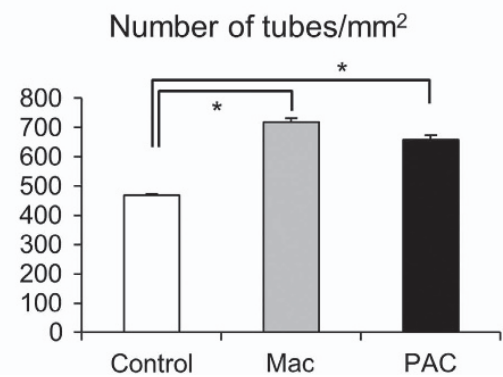

e

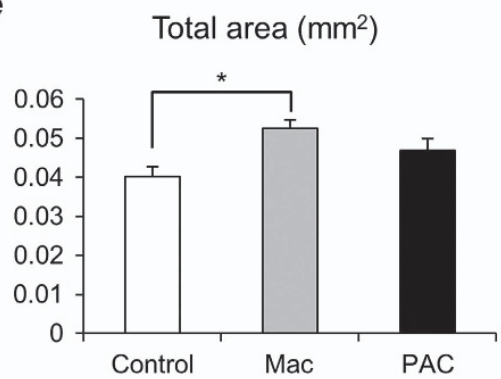

Figure $3 \mathrm{An}$ in vitro model of angiogenesis. (a) A schematic figure of an in vitro model of angiogenesis. Yellow fluorescent protein (YFP)-labeled human umbilical vein endothelial cells (HUVECs) were cultured on cyan fluorescent protein (CFP)-labeled mesenchymal stem cells (MSCs) in the presence of either red fluorescent protein (RFP)-labeled proangiogenic cells (PACs) or RFP-labeled macrophages (Macs). (b) After 7 days of culture, HUVECs formed vessel-like tubes on the MSC layer. (c) Representative figures of endothelial vessel-like tube formation of HUVECs for each group. (d) Both Macs and PACs significantly increased the number of tubes compared with the control ( $n=8$, respectively; Tukey's post hoc analysis - control vs Mac, $P=0.002$; control vs PAC, $P=0.016)$. * denotes statistical significance, $P<0.05$. (e) Only Macs significantly increased the total area of the tubes compared with the control $(n=8$, respectively; Tukey's post hoc analysis-control vs Mac, $P=0.018$ ).

growth factor, IL-17, basic fibroblast growth factor, platelet-derived growth factor-bb, vascular endothelial growth factor, IL-10 and tumor necrosis factor- $\alpha$ expression were not significantly different between PACs and Macs.

\section{PACs recruit more MSCs than do Macs in vitro}

Because the recruitment of pericytes or smooth muscle cells is necessary for neovascularization and vessel stabilization, we compared the interaction of PACs and Macs with MSCs in vitro. When we cultured MSCs covered with Matrigel, we observed a transmigration of MSCs through the Matrigel. PACs stimulated the transmigration of MSCs, leading to more MSC plaques in the Matrigel than there were when using Macs or PBS, suggesting that PACs stimulate chemotaxis in MSCs (Figure 2a, upper panel). We can quantify the total length of the transmigrated MSC network in the Matrigel (Figure 2a, 
a

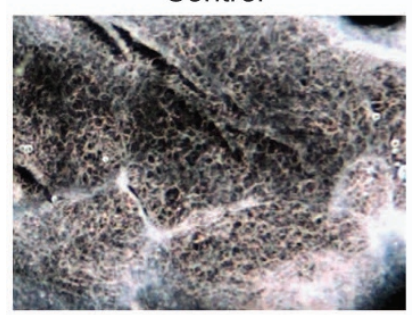

b

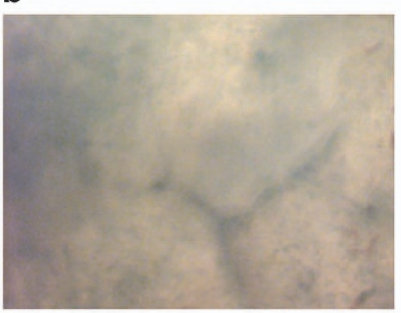

C

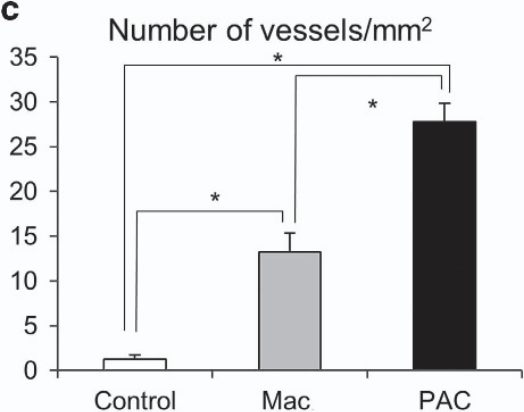

e

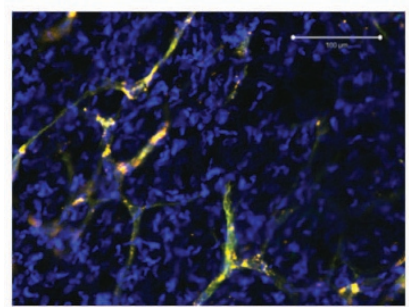

Mac
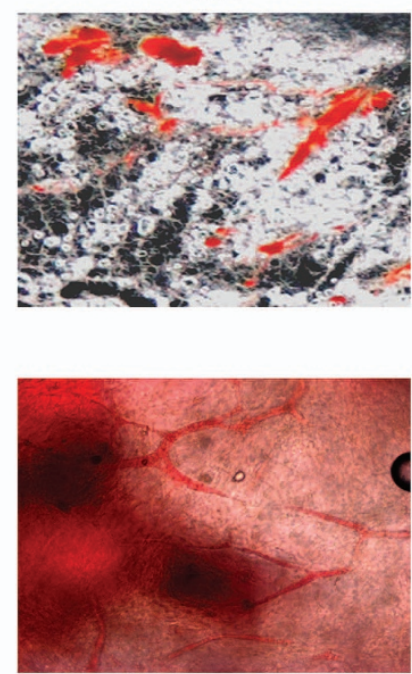

PAC
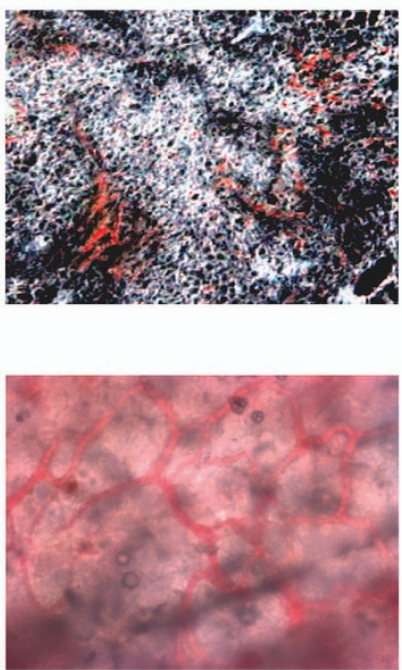

d

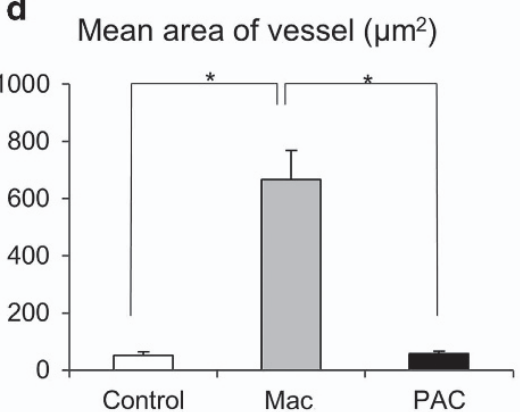

Control

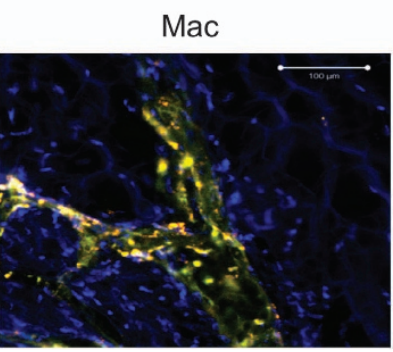

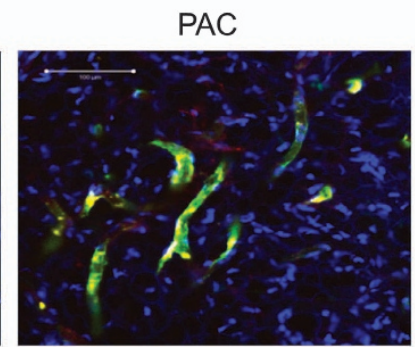

Figure 4 An in vivo model of angiogenesis. (a, b) Large and microscopic morphologies of thick sections (50 $\mu \mathrm{m})$ of in vivo Matrigel that was transplanted with human umbilical vein endothelial cells (HUVECs) and mesenchymal stem cells (MSCs) in the presence of either proangiogenic cells (PACs) or macrophages (Macs). Macs and PACs significantly increased blood perfusion into the Matrigel. Notably, Macs also increased hematoma formation in the Matrigel. (c) The density of vessels containing red blood cells in the Matrigel thick section. PACs significantly increased vessel density compared with those in the control and in the Mac-treated sections. Macs also increased vessel density compared with the control ( $n=9$, respectively, $P<0.001$ by analysis of variance (ANOVA)). Every pair is significant by Tukey's post hoc analysis. * denotes statistical significance, $P<0.05$. (d) The mean area of the vessels in Mac-transplanted Matrigel is significantly larger than in PAC-transplanted Matrigel or in the control $(n=9$, respectively, $P<0.001$ by ANOVA). Control vs Mac and Mac vs PAC were significant by Tukey's post hoc analysis. (e) Confocal microscopy revealed that the diameter of the vessels in the Mac group was larger than that in the PAC group or in the control.

lower panel). Stimulation by PACs significantly increased the total length of MSC transmigration compared with stimulation by PBS or Macs (Figure 2b).

As demonstrated above, PACs and Macs showed a small but significant difference in the characteristics of their surface markers, cytokines and MSC recruitment. To investigate whether there is difference between PACs and Macs in supporting vessel growth, we compared the effects of the presence of PACs and Macs in three different models of angiogenesis.

PACs and Macs enhanced two-dimensional pseudovascular tube formation in vitro

First, we co-cultured HUVECs and MSCs with PACs, Macs or a control with no cells after lentiviral transduction of yellow fluorescent protein, GFP and red fluorescent protein, 


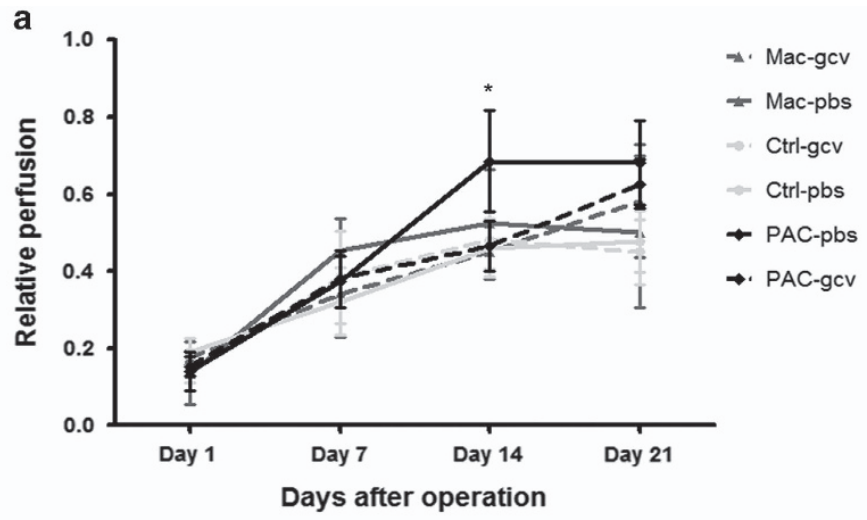

b

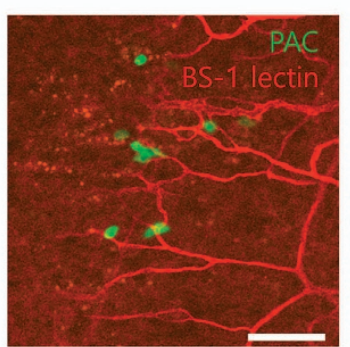

C

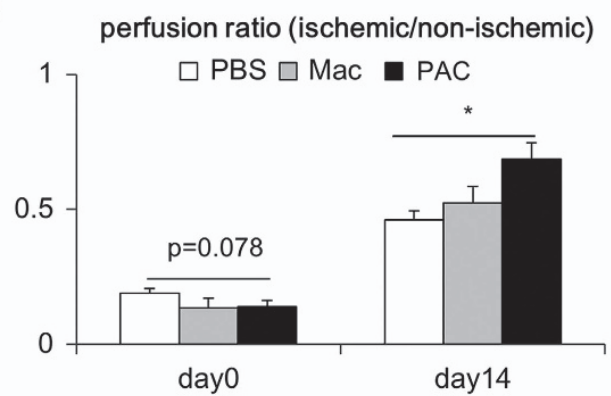

e
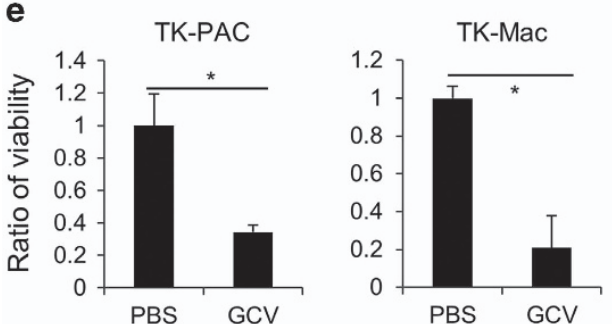

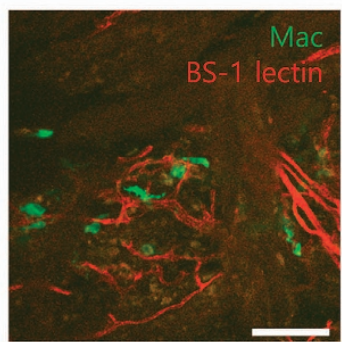

d

Capillary density $(/ \mathrm{mm} 2)$

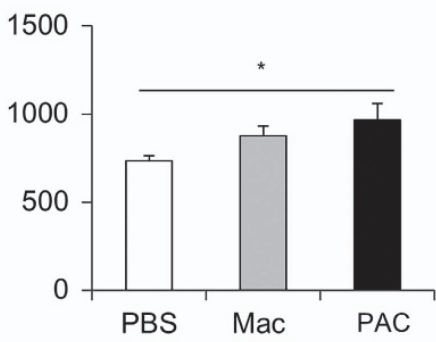

f

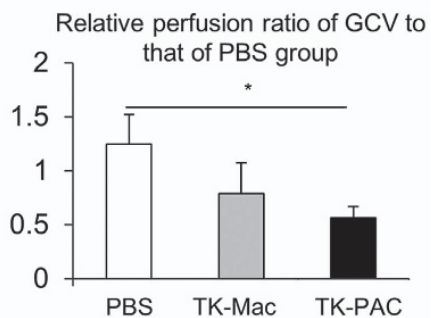

Figure 5 Proangiogenic cells (PACs) enhanced revascularization activity, and late depletion of PACs reversed the benefit in a hindlimb ischemia model. (a) Sequential measurement of relative perfusion in the ischemic limb by laser Doppler image analyses. * denotes statistical significance, $P<0.05$. (b) Green fluorescent protein (GFP)-labeled PACs or macrophages (Macs; green) were injected into the ischemic muscle. Robust cells were persistent in the ischemic muscle, particularly on the ischemic border zone (red: murine vessels, green: transplanted cells, scale bar: $100 \mu \mathrm{m})$. (c) Compared with the phosphate-buffered saline (PBS)-treated group, PACs significantly improved perfusion recovery to the ischemic limb at day 14 . However, the presence of Macs did not result in significant recovery relative to treatment with PBS ( $n=13,9$ and 12 in PBS, Mac and PAC, respectively. Each $P$-value was determined by one-way analysis of variance (ANOVA). For PAC vs PBS at day $14, P=0.003$ by Tukey's post hoc analysis). (d) Capillary density was significantly higher in the PAC-treated group at day 21 ( $n=8, P=0.05$ by the Kruskal-Wallis test; $P<0.05$ for PAC vs PBS by Tukey's post hoc analysis). (e) The MTT viability test shows that ganciclovir (GCV) incubation significantly decreases the survival of TK-PACs and TK-Macs relative to their survival after PBS incubation ( $n=4$, respectively). TK-Mac, TK-transduced Macs; TK-PAC, thymidine kinase-transduced PACs. (f) When GCV was injected into the mice at 7 days to 11 days after TK-PAC or TK-Mac transplantation, the perfusion of the TK-PAC-treated group deteriorated significantly compared with the PBS-treated group $(n=14,6$ and 18 in PBS, TK-Mac, and TK-PAC, respectively; $P=0.002$ by one-way ANOVA. For TK-PAC vs PBS, $P=0.001$ by Tukey's post hoc analysis). However, perfusion in the TK-Mac-treated group did not significantly change after GCV administration. 
respectively (Figure 3a). After 7 days, HUVECs formed capillary-like pseudovascular tubes on the MSC layer (Figure $3 \mathrm{~b}$ ). PACs and Macs were viable around the pseudovascular tubes for over a few weeks. When we quantified the pseudovascular tubes by yellow fluorescent protein signal, the presence of PACs and Macs was shown to have significantly increased the number of tubes compared with the control (Figures $3 \mathrm{c}$ and d). However, Macs but not PACs significantly increased the total area of the tubes compared with the control (Figure 3e).

PACs and Macs increased the number of perfused vessels in Matrigel in vivo, but only PACs supported vessel stability We transplanted growth factor-reduced Matrigels containing HUVECs and MSCEs with an equal number of PACs, Macs or a control with no cells into the subcutaneous pockets of nude mice. After 7 days, we harvested the Matrigel, and en-face images of the Matrigel clearly revealed blood-filled vessels lined with HUVECs, as we previously reported. ${ }^{16}$ When the Matrigel was sliced, blood-filled vessels were clearly visible in both celltreated groups, whereas the control group showed very few vessels in the Matrigel (Figure 4a). Under high magnification, the Matrigel that contained PACs supported an abundant formation of well-organized small vessels, whereas the presence of Macs induced disorganized large vessels that appeared to be unstable, leading to the formation of hematomas in the Matrigel (Figure 4b). The Matrigel plugs from the control group looked pale and gray when harvested because of low or no blood content inside. The number of blood-filled vessels was significantly higher in the PAC cotransplanted group than in the Mac group (Figure 4c). However, the mean vessel area (the total vessel area divided by the number of vessels) was significantly higher in Matrigels from the Mac group than in those from the PAC group, indicating that cotransplantation with Macs results in the formation of vessels with large diameters (Figure 4d).

Confocal microscopy revealed that the control group induced endothelial tube formation, although these tubes did not contain blood-filled lumens as in the Matrigel above (Figure 4e). However, the samples that contained Macs or PACs showed host vessel connections in the Matrigel (Supplementary Figure 1). The Mac group showed enlarged vessels with wider lumen diameters than those in the PAC group (Figure 4e).

\section{Evaluation of the contribution of PACs and Macs to neovascularization after hindlimb ischemia}

To determine the contribution of PACs and Macs to neovascularization after the induction of hindlimb ischemia, we injected PACs or Macs intravenously 1 day after the onset of ischemia. We analyzed the relative perfusion in the ischemic limb sequentially using laser Doppler images (Figure 5a). When the transplanted cells were transduced with lentiviral vectors expressing GFP and then injected into mice 1 day after the induction of hindlimb ischemia, GFP-labeled PACs and Macs were found incorporated at the border of the ischemic zone
(Figure 5b and Supplementary Figure 2). Interestingly, PACs were detected at a perivascular position around the vessels and were localized in the proximity of perfused and well-organized vessels, whereas Macs were often surrounded by enlarged vessels, similar to the in vivo findings we observed in the Matrigel assay. Although Macs did not enhance the recovery of the laser Doppler-derived blood flow 2 weeks after ischemia induction, PACs significantly increased the flow at 2 weeks after ischemia induction (Figure 5c). Capillary density, as measured 21 days after ischemia induction, was significantly higher in the PAC-treated mice than in the PBS-treated mice (Figure 5d). However, Mac-treated mice showed a statistically insignificant difference in capillary density from PBS-treated mice.

Finally, we evaluated the long-term effects of the presence of PACs or Macs on neovascularization by depleting the cells using an in vivo suicide gene approach, as we previously reported. ${ }^{16}$ We transduced PACs and Macs with a lentiviral vector encoding the TK gene that can activate the pro-apoptosis drug GCV to induce apoptosis in vivo. TK-transduced PACs (TK-PAC) and TK-transduced Macs (TK-Mac) were transduced with a similar efficiency, and $\sim 80 \%$ of the transduced cells became apoptotic by the addition of GCV in vitro (Figure 5e). Apoptosis in TK-transduced cells in vivo was induced by the daily injection of GCV from day 7 to day 11. Laser Doppler perfusion image analyses revealed that the relative blood flow to the ischemic limb was significantly reduced in the TK-PAC group, whereas the TK-Mac group showed only a minor reduction of blood flow recovery that was not significantly different from that in the control group (Figure 5f).

\section{DISCUSSION}

In the present study, although PACs and Macs shared some surface markers and secreted cytokines, they showed different expression profiles of angiogenic cytokines, different expression levels of some surface markers and different capabilities to recruit MSCs. The presence of Macs and PACs enhanced angiogenesis in an in vitro model, but Macs induced larger endothelial tubes than did PACs. In an in vivo Matrigel plug model, Macs induced disorganized and unstably enlarged vessels. In contrast, PACs induced well-organized perfused vessels. In the hindlimb ischemia model, we found that PAC transplantation significantly enhanced neovascularization activity. The depletion of PACs on day 7 abrogated this beneficial effect. In contrast, Mac transplantation did not improve the perfusion recovery of the ischemic limbs, and their depletion did not lead to a functional deterioration.

Various types of myeloid lineage cells, such as tumorassociated Macs, Tie2-expressing monocytes and myeloidderived suppressor cells, have been reported to have angiogenic capacities.22-24 The ex vivo cultured adult myeloid cells also demonstrated efficacy for therapeutic angiogenesis. ${ }^{4-6}$ All of the above myeloid lineage cells expressed monocytic and hematopoietic surface markers; however, they did not all enhance arteriogenesis or neovascularization. ${ }^{12,18-20}$ Therefore, 
the choice of one type of myeloid cell for therapeutic angiogenesis should be made with care because minor differences in the characterization of the two myeloid cells that were used in the present study were associated with a marked difference in the functional recovery and long-term outcome.

Macs induced angiogenesis via endothelial tube formation in vitro and vessel formation in the Matrigel model in vivo. However, Mac-induced angiogenesis was characterized by inappropriately dilated vessels; weak vascular stability, leading to hemorrhage or hematoma formation in the in vivo Matrigel; and insignificant in vivo functional recovery after hindlimb ischemia induction. This is similar to pathologic angiogenesis in tumors. Tumors frequently induce hypervascularization or abortive vessels. This process involves tumor-associated Macs and mast cells. ${ }^{25,26} \mathrm{M}$-CSF is known to induce the differentiation of monocyte lineage cells, promoting the formation of high-density vessel networks in tumors. ${ }^{27,28} \mathrm{M}$-CSF inhibition reduced the abundance of Macs, resulting in suppressed pathological neovascularization, but it did not affect the recovery of normal vasculature. ${ }^{29}$ Therefore, in the present study, Macs cultured in the presence of M-CSF might not show functional recovery in the ischemic hindlimb.

In contrast, PACs reproducibly induced healthy neovascularization. ${ }^{12,13}$ Although the mechanisms of healthy PAC-mediated angiogenesis and pathologic Mac-mediated angiogenesis were not thoroughly investigated in this study, we speculate that the following may be involved.

PACs and Macs showed rare endothelial differentiation. In the present study, they were preferentially localized to perivascular spaces, as was previously reported. ${ }^{10,11}$ The different cytokines used by PACs or Macs are suggested to have used paracrine mechanisms to mediate the difference in neovascularization that was found between these two cell types. Interestingly, PACs displayed a different cytokine expression profile than did Macs. Among the different cytokines, IL-8 was highly expressed in PACs. IL-8 was demonstrated to be a key angiogenic paracrine factor, and the blockade of IL- 8 but not of vascular endothelial growth factor prevented myeloid cellinduced angiogenesis. ${ }^{30}$ Some anti-angiogenic cytokines, such as angiopoietin 2 and angiostatin, were also differentially secreted by PACs. Anti-angiogenic cytokines regulate angiogenesis, and the absence of these cytokines leads to abnormal endothelial proliferation and vascular maturation. ${ }^{31-33}$ In addition, many clinical trials that tested single angiogenic factors to induce therapeutic angiogenesis failed to show a significant improvement, ${ }^{34}$ and an excess of angiogenic cytokines produced nonfunctioning vessels. ${ }^{35}$ Therefore, PACs seem to support healthy neovascularization activity not only by stimulating this process by angiogenic cytokines but also counterbalancing it by anti-angiogenic cytokines.

PACs were also better able to recruit MSCs in the Matrigel transmigration model. If PACs recruited mural cells to neovascularization sites, this may result in a more balanced and healthy revascularization of the ischemic organ. The recruitment of mural cells is a fundamental feature of vessel maturation and is known to be mediated by Transforming growth factor TGF-beta signaling, platelet-derived growth factor- $\beta$, and angiopoietin-1/Tie-2 signaling; ${ }^{36}$ however, the cytokine array assay revealed no significant differences in these cytokines between PACs and Macs. How PACs recruited more MSCs remains to be evaluated.

The balanced neovascularization that was mediated by PACs seemed to be effective for at least 7 days after transplantation because the depletion of PACs led to a decline in perfusion, whereas depletion of Macs did not affect perfusion, although both cell types persisted over 7 days after transplantation. This fact suggests that the presence of PACs not only increases vessel density but also supports the maturation of the newly formed vessels, leading to enhanced perfusion. Long-term effects may be mediated by the interaction between PACs and endothelial tip cells, leading to the bridging of tip cells from different vessel segments and to an increase in the fusion of endothelial tubes at neovascularization sites. ${ }^{19}$ The fact that we frequently observed PACs at the fronts of angiogenic spouts in the ischemic hindlimb, in addition to the finding that PACs improved blood perfusion into the transplanted Matrigel in vivo, strongly supports the role of PACs as cellular chaperones for vascular anastomosis and maturation.

In the present study, we compared the angiogenic properties of PACs with those of authentic Macs in different experimental models of angiogenesis to gain further insights into the mechanisms of PAC-mediated angiogenesis. In conclusion, long-term persistence and healthy neovascularization seem to be unique to PACs and are probably mediated by balanced cytokines and by the recruitment of mural cells.

\section{ACKNOWLEDGEMENTS}

The project was supported by the Basic Science Research Program through the National Research Foundation of Korea (NRF) that is funded by the Ministry of Education, Science and Technology (2012R1A2A2A02012821 and 2012M3A9C7050140), and by Grant No. 03-2013-011 from the SNUBH Research Fund.

1 Asahara T, Murohara T, Sullivan A, Silver M, van der Zee R, Li T et al. Isolation of putative progenitor endothelial cells for angiogenesis. Science 1997; 275: 964-967.

2 Kawamoto A, Tkebuchava T, Yamaguchi J, Nishimura H, Yoon YS Milliken $\mathrm{C}$ et al. Intramyocardial transplantation of autologous endothelial progenitor cells for therapeutic neovascularization of myocardial ischemia. Circulation 2003; 107: 461-468.

3 Kang HJ, Kim HS, Zhang SY, Park KW, Cho HJ, Koo BK et al. Effects of intracoronary infusion of peripheral blood stem-cells mobilised with granulocyte-colony stimulating factor on left ventricular systolic function and restenosis after coronary stenting in myocardial infarction: the MAGIC cell randomised clinical trial. Lancet 2004; 363: 751-756.

4 Kalka C, Masuda H, Takahashi T, Kalka-Moll WM, Silver M, Kearney M et al. Transplantation of ex vivo expanded endothelial progenitor cells for therapeutic neovascularization. Proc Natl Acad Sci USA 2000; 97: 3422-3427.

5 Assmus B, Honold J, Schachinger V, Britten MB, Fischer-Rasokat U, Lehmann $\mathrm{R}$ et al. Transcoronary transplantation of progenitor cells after myocardial infarction. N Engl J Med 2006; 355: 1222-1232.

6 Hur J, Yoon CH, Kim HS, Choi JH, Kang HJ, Hwang KK et al. Characterization of two types of endothelial progenitor cells and their different contributions to neovasculogenesis. Arterioscler Thromb Vasc Biol 2004; 24: 288-293. 
7 Gulati R, Jevremovic D, Peterson TE, Chatterjee S, Shah V, Vile RG et al. Diverse origin and function of cells with endothelial phenotype obtained from adult human blood. Circ Res 2003; 93: 1023-1025.

8 Rehman J, Li J, Orschell CM, March KL. Peripheral blood "endothelial progenitor cells" are derived from monocyte/macrophages and secrete angiogenic growth factors. Circulation 2003; 107: 1164-1169.

9 Hur J, Yoon CH, Lee CS, Kim TY, Oh IY, Park KW et al. Akt is a key modulator of endothelial progenitor cell trafficking in ischemic muscle. Stem Cells 2007; 25: 1769-1778.

10 Ziegelhoeffer T, Fernandez B, Kostin S, Heil M, Voswinckel R, Helisch A et al. Bone marrow-derived cells do not incorporate into the adult growing vasculature. Circ Res 2004; 94: 230-238.

11 Zentilin L, Tafuro S, Zacchigna S, Arsic N, Pattarini L, Sinigaglia M et al. Bone marrow mononuclear cells are recruited to the sites of VEGF-induced neovascularization but are not incorporated into the newly formed vessels. Blood 2006; 107: 3546-3554.

12 Yoon CH, Hur J, Park KW, Kim JH, Lee CS, Oh IY et al. Synergistic neovascularization by mixed transplantation of early endothelial progenitor cells and late outgrowth endothelial cells: the role of angiogenic cytokines and matrix metalloproteinases. Circulation 2005; 112: 1618-1627.

13 Urbich C, Aicher A, Heeschen C, Dernbach E, Hofmann WK, Zeiher AM et al. Soluble factors released by endothelial progenitor cells promote migration of endothelial cells and cardiac resident progenitor cells. J Mol Cell Cardiol 2005; 39: 733-742.

14 Cho HJ, Lee N, Lee JY, Choi YJ, li M, Wecker A et al. Role of host tissues for sustained humoral effects after endothelial progenitor cell transplantation into the ischemic heart. J Exp Med 2007; 204: 3257-3269

15 Ziebart T, Yoon CH, Trepels T, Wietelmann A, Braun T, Kiessling F et al. Sustained persistence of transplanted proangiogenic cells contributes to neovascularization and cardiac function after ischemia. Circ Res 2008; 103: 1327-1334

16 Yoon CH, Koyanagi M, lekushi K, Seeger F, Urbich C, Zeiher AM et al. Mechanism of improved cardiac function after bone marrow mononuclear cell therapy: role of cardiovascular lineage commitment. Circulation 2010; 121: 2001-2011.

17 Hashimoto S, Yamada M, Motoyoshi K, Akagawa KS. Enhancement of macrophage colony-stimulating factor-induced growth and differentiation of human monocytes by interleukin-10. Blood 1997; 89: 315-321.

18 Arras M, Ito WD, Scholz D, Winkler B, Schaper J, Schaper W. Monocyte activation in angiogenesis and collateral growth in the rabbit hindlimb. J Clin Invest 1998; 101: 40-50.

19 Fantin A, Vieira JM, Gestri G, Denti L, Schwarz Q, Prykhozhij S et al. Tissue macrophages act as cellular chaperones for vascular anastomosis downstream of VEGF-mediated endothelial tip cell induction. Blood 2010; 116: 829-840.

20 Urbich C, Heeschen C, Aicher A, Dernbach E, Zeiher AM, Dimmeler S. Relevance of monocytic features for neovascularization capacity of circulating endothelial progenitor cells. Circulation 2003; 108: 2511-2516.

21 Yoon CH, Choi YE, Koh SJ, Choi JI, Park YB, Kim HS. High glucoseinduced jagged 1 in endothelial cells disturbs notch signaling for angiogenesis: a novel mechanism of diabetic vasculopathy. J Mol Cell Cardiol 2014; 69: 52-66.

22 Lewis CE, Pollard JW. Distinct role of macrophages in different tumor microenvironments. Cancer Res 2006; 66: 605-612.
23 Ostrand-Rosenberg S, Sinha P. Myeloid-derived suppressor cells: linking inflammation and cancer. J Immunol 2009; 182: 4499-4506.

24 Venneri MA, De Palma M, Ponzoni M, Pucci F, Scielzo C, Zonari E et al. Identification of proangiogenic TIE2-expressing monocytes (TEMs) in human peripheral blood and cancer. Blood 2007; 109: 5276-5285.

25 Coussens LM, Raymond WW, Bergers G, Laig-Webster M, Behrendtsen O, Werb $\mathrm{Z}$ et al. Inflammatory mast cells up-regulate angiogenesis during squamous epithelial carcinogenesis. Genes Dev 1999; 13: 1382-1397.

26 Sica A, Saccani A, Mantovani A. Tumor-associated macrophages: a molecular perspective. Int Immunopharmacol 2002; 2: 1045-1054.

27 Curry JM, Eubank TD, Roberts RD, Wang Y, Pore N, Maity A et al. M-CSF signals through the MAPK/ERK pathway via Sp1 to induce VEGF production and induces angiogenesis in vivo. PLoS One 2008; 3 : e3405.

28 Okazaki T, Ebihara S, Takahashi H, Asada M, Kanda A, Sasaki H. Macrophage colony-stimulating factor induces vascular endothelial growth factor production in skeletal muscle and promotes tumor angiogenesis. $\mathrm{J}$ Immunol 2005; 174: 7531-7538.

29 Kubota Y, Takubo K, Shimizu T, Ohno H, Kishi K, Shibuya M et al. M-CSF inhibition selectively targets pathological angiogenesis and lymphangiogenesis. J Exp Med 2009; 206: 1089-1102.

30 Medina RJ, O'Neill CL, O'Doherty TM, Knott H, Guduric-Fuchs J, Gardiner TA et al. Myeloid angiogenic cells act as alternative M2 macrophages and modulate angiogenesis through interleukin-8. Mol Med 2011; 17: $1045-1055$.

31 O'Reilly MS, Boehm T, Shing Y, Fukai N, Vasios G, Lane WS et al. Endostatin: an endogenous inhibitor of angiogenesis and tumor growth. Cell 1997; 88: 277-285.

32 O'Reilly MS, Holmgren L, Shing Y, Chen C, Rosenthal RA, Moses M et al. Angiostatin: a novel angiogenesis inhibitor that mediates the suppression of metastases by a Lewis lung carcinoma. Cell 1994; 79: 315-328.

33 Feng $Y$, Vom Hagen F, Wang Y, Beck S, Schreiter K, Pfister F et al. The absence of angiopoietin-2 leads to abnormal vascular maturation and persistent proliferative retinopathy. Thromb Haemost 2009; 102 : 120-130.

34 Freedman SB, Isner JM. Therapeutic angiogenesis for ischemic cardiovascular disease. J Mol Cell Cardiol 2001; 33: 379-393.

35 Dor Y, Djonov V, Abramovitch R, Itin A, Fishman GI, Carmeliet P et al. Conditional switching of VEGF provides new insights into adult neovascularization and pro-angiogenic therapy. EMBO J 2002; 21: 1939-1947.

36 Potente M, Gerhardt H, Carmeliet P. Basic and therapeutic aspects of angiogenesis. Cell 2011; 146: 873-887.

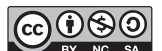

This work is licensed under a Creative Commons Attribution-NonCommercial-ShareAlike 4.0 International License. The images or other third party material in this article are included in the article's Creative Commons license, unless indicated otherwise in the credit line; if the material is not included under the Creative Commons license, users will need to obtain permission from the license holder to reproduce the material. To view a copy of this license, visit http:// creativecommons.org/licenses/by-nc-sa/4.0/

Supplementary Information accompanies the paper on Experimental \& Molecular Medicine website (http://www.nature.com/emm) 